\title{
RESIDUAL QUOTIENT FUZZY SUBSET IN NEAR-RINGS
}

\author{
P. DHEENA AND S. COUMARESSANE
}

Received 27 March 2006; Revised 14 August 2006; Accepted 17 October 2006

For any fuzzy subsets $\lambda$ and $\mu$, we introduce the notion of residual quotient fuzzy subset $(\lambda: \mu)$ and we have characterized residual quotient fuzzy subset in near-rings.

Copyright (c) 2006 Hindawi Publishing Corporation. All rights reserved.

\section{Introduction}

In 1965, Zadeh [14] introduced the concept of fuzzy subsets and studied their properties on the parallel lines to set theory. In 1971, Rosenfeld [10] defined the fuzzy subgroup and gave some of its properties. Rosenfeld's definition of a fuzzy group is a turning point for pure mathematicians. Since then, the study of fuzzy algebraic structure has been pursued in many directions such as groups, rings, modules, vector spaces, and so on. In 1981, Das [2] explained the interrelationship between the fuzzy subgroups and their $t$-level subsets. Fuzzy subrings and ideals were first introduced by Wang-jin Liu [5] in 1982. Subsequently, Mukherjee and Sen [7], Swamy and Swamy [13], Dixit et al. [3], and Rajesh Kumar [4] applied some basic concepts pertaining to ideals from classical ring theory and developed a theory of fuzzy. The notions of fuzzy subnear-ring and ideal were first introduced by Abou-Zaid [1] in 1991.

Wang-jin Liu [6] introduced residual quotient fuzzy subset $(\lambda: \mu)$ for any two fuzzy ideals in rings. In this paper, we introduce residual quotient fuzzy subset $(\lambda: \mu)$ for any two fuzzy subsets, which is different from [6], and we characterize some related results in near-rings.

\section{Preliminaries}

We would like to reproduce some definitions and results proposed by the pioneers in this field earlier for the sake of completeness.

Definition 2.1. A near-ring $N$ is a system with two binary operations + and $\cdot$ such that

(1) $(N,+)$ is a group, not necessarily abelian;

(2) $(N, \cdot)$ is a semigroup;

(3) $(x+y) z=x z+y z$ for all $x, y, z \in N$. 
We will use the word "near-ring" to mean "right distributive near-ring." We denote $x y$ instead of $x \cdot y$. Note that $0 \cdot x=0$ and $(-x) y=-x y$ but in general $x \cdot 0 \neq 0$ for some $x \in N$.

Definition 2.2. Let $(N,+, \cdot)$ be a near-ring. A subset $I$ of $N$ is said to be an ideal of $N$ if

(1) $(I,+)$ is a normal subgroup of $(N,+)$;

(2) $I N \subseteq I$;

(3) $n_{1}\left(n_{2}+i\right)-n_{1} n_{2} \in I$ for all $i \in I$ and $n_{1}, n_{2} \in N$.

If $I$ satisfies (1) and (2), then it is called a right ideal of $N$. If $I$ satisfies (1) and (3), then it is called a left ideal of $N$.

Let $N$ be a near-ring. Given two subsets $A$ and $B$ of $N$, the product $A B=\{a b \mid a \in$ $A, b \in B\}$ and $A * B=\left\{a\left(a^{\prime}+b\right)-a a^{\prime} \mid a, a^{\prime} \in A, b \in B\right\}$. From now on, throughout this paper $N$ will denote right distributive near-ring, unless otherwise specified. For the basic terminology and notation, we refer to Pilz [9] and Abou-Zaid [1].

Definition 2.3. A mapping $\mu: N \rightarrow[0,1]$ is called a fuzzy subset of $N$.

A fuzzy subset $\mu: N \rightarrow[0,1]$ is nonempty if $\mu$ is not the constant map which assumes the value 0 . For any two fuzzy subsets $\lambda$ and $\mu$ of $N, \lambda \leq \mu$ means that $\lambda(a) \leq \mu(a)$ for all $a \in N$. The characteristic function of $N$ is denoted by $\mathbf{N}$ and, of its subset $A$ is denoted by $f_{A}$. The image of a fuzzy subset $\mu$ is denoted by $\operatorname{Im}(\mu)=\{\mu(n) \mid n \in N\}$. Hereafter, we consider only nonempty fuzzy subsets of $N$.

Definition 2.4. Let $E$ be an $N$-group and let $\mu$ be a fuzzy subset of $E$. Then $\mu$ is called a fuzzy $N$-subgroup of $N$ if for all $x, y \in E$ and $n \in N$,

(1) $\mu(x-y) \geq \min \{\mu(x), \mu(y)\}$;

(2) $\mu(n x) \geq \mu(x)$.

Definition 2.5. Let $\mu$ be any fuzzy subset of $N$. For $t \in[0,1]$, the set $\mu_{t}=\{x \in N \mid \mu(x) \geq t\}$ is called a level subset of $\mu$.

Definition 2.6. Let $f$ and $g$ be any two fuzzy subsets of $N$. Then $f \cap g, f \cup g, f+g, f g$, and $f * g$ are fuzzy subsets of $N$ defined by

$$
\begin{aligned}
& (f \cap g)(x)=\min \{f(x), g(x)\}, \\
& (f \cup g)(x)=\max \{f(x), g(x)\}, \\
& (f+g)(x)= \begin{cases}\sup _{x=y+z}\{\min \{f(y), g(z)\}\}, & \text { if } x \text { is expressed as } x=y+z, \\
0, & \text { otherwise, }\end{cases} \\
& (f g)(x)= \begin{cases}\sup _{x=y z}\{\min \{f(y), g(z)\}\}, & \text { if } x \text { is expressed as } x=y z, \\
0, & \text { otherwise }\end{cases} \\
& (f * g)(x)= \begin{cases}\sup _{x=a(b+c)-a b}\{\min \{f(a), g(c)\}\}, & \text { if } x=a(b+c)-a b, \\
0, & \text { otherwise. }\end{cases}
\end{aligned}
$$


Definition 2.7. For any $x \in N$ and $t \in(0,1]$, define a fuzzy point $x_{t}$ as

$$
x_{t}(y)= \begin{cases}t, & \text { if } y=x, \\ 0, & \text { if } y \neq x\end{cases}
$$

If $x_{t}$ is a fuzzy point and $\mu$ is any fuzzy subset of $N$ and $x_{t} \leq \mu$, then we write $x_{t} \in \mu$. Note that $x_{t} \in \mu$ if and only if $x \in \mu_{t}$ where $\mu_{t}$ is a level subset of $\mu$. If $x_{r}$ and $y_{s}$ are fuzzy points, then $x_{r} y_{s}=(x y)_{\min \{r, s\}}$.

Definition 2.8. Let $\mu$ be a nonempty fuzzy subset of $N . \mu$ is a fuzzy ideal of $N$ if for all $x, y, i \in N$,

(1) $\mu(x-y) \geq \min \{\mu(x), \mu(y)\}$;

(2) $\mu(x+y)=\mu(y+x)$;

(3) $\mu(x y) \geq \mu(x)$;

(4) $\mu(x(y+i)-x y) \geq \mu(i)$.

If $\mu$ satisfies (1), (2), and (3), then it is called a fuzzy right ideal of $N$. If $\mu$ satisfies (1), (2), and (4), then it is called a fuzzy left ideal of $N$. If $\mu$ is both fuzzy right and fuzzy left ideal of $N$, then $\mu$ is called a fuzzy ideal of $N$.

Definition 2.9. An ideal $P$ of $N$ is called completely prime if any two elements $a, b$ of $N$ such that $a b \in P$ implies either $a \in P$ or $b \in P$.

Definition 2.10. An ideal $P$ of $N$ is called completely semiprime if any element $a$ of $N$ such that $a^{2} \in P$ implies $a \in P$ for all $x \in N$.

Definition 2.11. A fuzzy ideal $\mu$ of $N$ is called completely fuzzy prime ideal if any two fuzzy points $x_{r}, y_{s}$ of $N$ such that $x_{r} y_{s} \in \mu$ implies either $x_{r} \in \mu$ or $y_{s} \in \mu$ for all $x, y \in N$ and for all $r, s \in[0,1]$.

Definition 2.12. A fuzzy ideal $\mu$ of $N$ is called completely fuzzy semiprime ideal if any fuzzy point $x_{r}$ of $N$ such that $\left(x_{r}\right)^{2} \in \mu$ implies $x_{r} \in \mu$ for all $x \in N$ and $r \in[0,1]$.

LEMMA 2.13 [11]. Let I be a nonempty subset of $N$. I is an $N$-subgroup of $N$ if and only if $f_{I}$ is a fuzzy $N$-subgroup of $N$.

Lemma 2.14 [11]. Let $\mu$ be a fuzzy subset of $N$. $\mu$ is a fuzzy $N$-subgroup of $N$ if and only if the level subset $\mu_{t}, t \in \operatorname{Im}(\mu)$, is an $N$-subgroup of $N$.

Lемма 2.15 [1]. Let I be a subset of $N$. I is an (left or right) ideal of $N$ if and only if $f_{I}$ is a fuzzy (left or right) ideal of $N$.

Lemma 2.16 [1]. Let $\mu$ be a fuzzy subset of $N$. $\mu$ is a fuzzy (left or right) ideal of $N$ if and only if the level subset $\mu_{t}, t \in \operatorname{Im}(\mu)$, is an ideal of $N$.

Definition 2.17. A subgroup $Q$ of $(N,+)$ is said to be a quasi-ideal of $N$ if $Q N \cap N Q \cap$ $N * Q \subseteq Q$.

A subgroup $B$ of $(N,+)$ is said to be a bi-ideal of $N$ if $B N B \cap(B N) * B \subseteq B$.

Now we introduce the notion of fuzzy bi-ideal of $N$. We characterize fuzzy quasi-ideal and fuzzy bi-ideal of $N$. 
4 Residual quotient fuzzy subset in near-rings

Definition 2.18 [8]. A fuzzy subgroup $\mu$ of $N$ is called a fuzzy quasi-ideal of $N$ if $(\mu \mathbf{N}) \cap$ $(\mathbf{N} \mu) \cap(\mathbf{N} * \mu) \leq \mu$.

Definition 2.19. A fuzzy subgroup $\mu$ of $N$ is called a fuzzy bi-ideal of $N$ if $(\mu \mathbf{N} \mu) \cap(\mu \mathbf{N} *$ $\mu) \leq \mu$.

Note that

$$
\begin{aligned}
(\mu \mathbf{N} * \mu)(z) & =\sup _{z=x(y+c)-x y}\{\min \{(\mu \mathbf{N})(x), \mu(c)\}\} \\
& =\sup _{z=x(y+c)-x y}\left\{\min \left\{\sup _{x=x_{1} x_{2}}\left\{\mu\left(x_{1}\right), \mu(c)\right\}\right\}\right\} \\
& =\sup _{z=x_{1} x_{2}(y+c)-x_{1} x_{2} y}\left\{\min \left\{\mu\left(x_{1}\right), \mu(c)\right\}\right\} \\
& =0, \quad \text { otherwise. }
\end{aligned}
$$

It is very clear that if $N$ is a zero-symmetric near-ring, then $\mu \mathrm{N} \mu \leq \mu$ for every fuzzy bi-ideal $\mu$.

Lemma 2.20 [8]. Let $\mu$ be a fuzzy subset of $N$. If $\mu$ is a fuzzy left ideal (right ideal, $N$ subgroup, subnear-ring) of $N$, then $\mu$ is a fuzzy quasi-ideal of $N$.

Proof. Let $\mu$ be a fuzzy left ideal of $N$. Let $x \in N$ and $x=a b=n_{1}\left(n_{2}+c\right)-n_{1} n_{2}$, where $a$, $b, n_{1}, n_{2}$, and $c$ are in $N$. Consider

$$
\begin{aligned}
(\mu \mathbf{N} \cap & \mathbf{N} \mu \cap \mathbf{N} * \mu)(x) \\
= & \min \{(\mu \mathbf{N})(x),(\mathbf{N} \mu)(x),(\mathbf{N} * \mu)(x)\} \\
= & \min \left\{\sup _{x=a b}\{\mu(a)\}, \sup _{x=a b}\{\mu(b)\}, \sup _{\left.x=n_{1}\left(n_{2}+c\right)-n_{1} n_{2}\right)}\{\mu(c)\}\right\} \\
\leq & \min \left\{1,1, \sup _{\left.x=n_{1}\left(n_{2}+c\right)-n_{1} n_{2}\right)}\left\{\mu\left(n_{1}\left(n_{2}+c\right)-n_{1} n_{2}\right)\right\}\right\} \\
& \left(\text { as } \mu \text { is a fuzzy left ideal, } \mu\left(n_{1}\left(n_{2}+c\right)-n_{1} n_{2}\right) \geq \mu(c)\right) \\
\leq & \mu(x) .
\end{aligned}
$$

We remark that if $x$ is not expressed as $x=a b=n_{1}\left(n_{2}+c\right)-n_{1} n_{2}$, then $(\mu \mathbf{N} \cap \mathbf{N} \mu \cap$ $\mathbf{N} * \mu)(x)=0 \leq \mu(x)$. Thus $\mu \mathbf{N} \cap \mathbf{N} \mu \cap \mathbf{N} * \mu \leq \mu$. Hence $\mu$ is a fuzzy quasi-ideal of $N$.

Lemma 2.21. For any nonempty subsets $A$ and $B$ of $N$,

(1) $f_{A} f_{B}=f_{A B}$;

(2) $f_{A} \cap f_{B}=f_{A \cap B}$;

(3) $f_{A} * f_{B}=f_{A * B}$.

Proof. Proof is straight forward. 
LEMMA 2.22. Let $Q$ be a subgroup of $N$.

(1) $Q$ is a quasi-ideal of $N$ if and only if $f_{Q}$ is a fuzzy quasi-ideal of $N$.

(2) $Q$ is a bi-ideal of $N$ if and only if $f_{Q}$ is a fuzzy bi-ideal of $N$.

Proof. Proof of (1) can easily be seen in [8].

Proof of (2). Assume that $Q$ is a bi-ideal of $N$. Then $f_{Q}$ is a fuzzy subgroup of $N . f_{Q} f_{N} f_{Q} \cap$ $f_{Q} f_{N} * f_{Q} \leq f_{Q N Q \cap Q N * Q} \leq f_{Q}$. This means that $f_{Q}$ is a fuzzy bi-ideal of $N$.

Conversely, let us assume that $f_{Q}$ is a fuzzy quasi-ideal of $N$. Let $y$ be any element of $Q N Q \cap Q N * Q$. Then, we have

$$
f_{Q}(y) \geq\left(f_{Q} f_{N} f_{Q} \cap f_{Q} f_{N} * f_{B}\right)(y)=f_{Q N Q \cap Q N * Q}(y)=1
$$

Thus $y \in Q$ and $Q N Q \cap Q N * Q \subseteq Q$. Hence $Q$ is a bi-ideal of $N$.

Lemma 2.23. Any fuzzy quasi-ideal of $N$ is a fuzzy bi-ideal of $N$.

Proof. Let $\mu$ be any fuzzy quasi-ideal of $N$. Then, we have

$$
\begin{gathered}
\mu \mathbf{N} \mu \subseteq \mu(\mathbf{N} \mathbf{N}) \subseteq \mu \mathbf{N}, \\
\mu \mathbf{N} \mu \subseteq(\mathbf{N} \mathbf{N}) \mu \subseteq \mathbf{N} \mu, \\
\mu \mathbf{N} * \mu \subseteq(\mathbf{N} \mathbf{N}) * \mu \subseteq \mathbf{N} * \mu, \\
\mu \mathbf{N} \mu \cap \mu \mathbf{N} * \mu \subseteq \mu \mathbf{N} \cap \mathbf{N} \mu \cap \mathbf{N} * \mu \subseteq \mu .
\end{gathered}
$$

Hence, $\mu$ is a fuzzy bi-ideal of $N$.

However, the converse of Lemma 2.23 is not true.

Example 2.24. Let $N=\{0, a, b, c\}$ be Klein's four group. Define multiplication in $N$ as follows:

\begin{tabular}{c|cccc}
\hline+ & 0 & $\mathrm{a}$ & $\mathrm{b}$ & $\mathrm{c}$ \\
\hline 0 & 0 & $\mathrm{a}$ & $\mathrm{b}$ & $\mathrm{c}$ \\
$\mathrm{a}$ & $\mathrm{a}$ & 0 & $\mathrm{c}$ & $\mathrm{b}$ \\
$\mathrm{b}$ & $\mathrm{b}$ & $\mathrm{c}$ & 0 & $\mathrm{a}$ \\
$\mathrm{c}$ & $\mathrm{c}$ & $\mathrm{b}$ & $\mathrm{a}$ & 0 \\
\hline
\end{tabular}

\begin{tabular}{c|cccc}
\hline$\bullet$ & 0 & $\mathrm{a}$ & $\mathrm{b}$ & $\mathrm{c}$ \\
\hline 0 & 0 & 0 & 0 & 0 \\
$\mathrm{a}$ & 0 & $\mathrm{~b}$ & 0 & $\mathrm{~b}$ \\
$\mathrm{~b}$ & 0 & 0 & 0 & 0 \\
$\mathrm{c}$ & 0 & $\mathrm{~b}$ & 0 & $\mathrm{~b}$ \\
\hline
\end{tabular}

Then, $(N,+, \bullet)$ is a near-ring (see [9, Page 408, Scheme 15]).

Define $\mu: N \rightarrow[0,1]$ by

$$
\mu(x)= \begin{cases}1, & \text { if } x=0, a \\ 0, & \text { otherwise }\end{cases}
$$


6 Residual quotient fuzzy subset in near-rings

For any $t \in[0,1], \mu_{t}=\{0, a\}$ or $\{0, a, b, c\}$. Since $\{0, a\}$ and $\{0, a, b, c\}$ are bi-ideal in $N, \mu_{t}$ is the bi-ideal in $N$ for all $t$. Hence $\mu$ is a fuzzy bi-ideal of $N$. Now,

$$
\begin{aligned}
(\mu \mathbf{N})(b)= & \sup _{b=x y}\{\min \{\mu(x), \mathbf{N}(y)\}\} \\
= & \sup \{\min \{\mu(a), \mathbf{N}(a)\}, \min \{\mu(c), \mathbf{N}(c)\}, \min \{\mu(a), \mathbf{N}(c)\}, \\
& \quad \min \{\mu(c), \mathbf{N}(a)\}\}, \quad \text { as } b=a \cdot a=c \cdot c=a \cdot c=c \cdot a, \\
= & \sup \{\min \{1,1\}, \min \{0,1\}, \min \{1,1\}, \min \{0,1\}\} \\
= & \sup \{1,0,1,0\} \\
= & 1 .
\end{aligned}
$$

Similarly, we have $(\mathbf{N} \mu)(b)=1$. Thus,

$$
(\mu \mathbf{N} \cap \mathbf{N} \mu)(b)=\min \{(\mu \mathbf{N})(b),(\mathbf{N} \mu)(b)\}=\min \{1,1\}=1 .
$$

But $\mu(b)=0$. Thus, $(\mu \mathbf{N} \cap \mathbf{N})(b)=1 \nless \mu(b)=0$. Therefore, $\mu$ is not a fuzzy quasi-ideal of $N$.

Lemma 2.25. Let $\mu$ be a fuzzy subset of $N$. If $\mu$ is a fuzzy left ideal (right ideal, $N$-subgroup, subnear-ring) of $N$, then $\mu$ is a fuzzy bi-ideal of $N$.

Proof. As $\mu$ is a left ideal of $N$ and Lemma 2.20, $\mu$ is a fuzzy quasi-ideal of $N$. Hence by Lemma 2.23, $\mu$ is a fuzzy bi-ideal of $N$.

Theorem 2.26. Let $\mu$ be a fuzzy subset of $N$. If $\mu$ is a fuzzy quasi-ideal of $N$, if and only if $\mu_{t}$ is a quasi-ideal of $N$, for all $t \in \operatorname{Im}(\mu)$.

Proof. Let $\mu$ be a fuzzy quasi-ideal of $N$. Let $t \in \operatorname{Im}(\mu)$. Suppose $x, y \in N$ such that $x, y \in$ $\mu_{t}$. Then $\mu(x) \geq t, \mu(y) \geq t$, and $\min \{\mu(x), \mu(y)\} \geq t$. As $\mu$ is a fuzzy quasi-ideal, $\mu(x-$ $y) \geq t$ and hence $x-y \in \mu_{t}$. Let $x \in N$. Suppose $x \in \mu_{t} N \cap N \mu_{t} \cap N * \mu_{t}$. Then, there exist $a, b, c \in \mu_{t}$ and $n_{1}, n_{2}, n_{3}, n_{4} \in N$ such that $x=a n_{1}=n_{2} b=n_{3}\left(n_{4}+c\right)-n_{3} n_{4}$. Thus, $\mu(a) \geq t, \mu(b) \geq t$, and $\mu(c) \geq t$. Then,

$$
\begin{aligned}
(\mu \mathbf{N} \cap \mathbf{N} \mu \cap \mathbf{N} * \mu)(x) & =\min \{(\mu \mathbf{N})(x),(\mathbf{N} \mu)(x),(\mathbf{N} * \mu)(x)\} \\
& =\min \left\{\sup _{x=a n_{1}} \mu(a), \sup _{x=n_{2} b} \mu(b), \sup _{x=n_{3}\left(n_{4}+c\right)-n_{3} n_{4}} \mu(c)\right\} \\
& \geq t .
\end{aligned}
$$

As $\mu$ is the fuzzy quasi-ideal of $N, \mu(x) \geq t$. Thus, $x \in \mu_{t}$ and hence $\mu_{t}$ is a quasi-ideal of $N$. 
Conversely, let us assume that $\mu_{t}, t \in \operatorname{Im}(\mu)$, is a quasi-ideal of $N$. Let $x \in N$. Consider

$$
\begin{aligned}
(\mu \mathbf{N} \cap \mathbf{N} \mu \cap \mathbf{N} * \mu)(x) & =\min \{(\mu \mathbf{N})(x),(\mathbf{N} \mu)(x),(\mathbf{N} * \mu)(x)\} \\
& =\min \left\{\sup _{x=a b}\{\min \{\mu(a), \mathbf{N}(b)\}\}, \sup _{x=a b}\{\min \{\mathbf{N}(a), \mu(b)\}\},\right. \\
& =\min \left\{\sup _{x=n_{1}\left(n_{2}+c\right)-n_{1} n_{2}}\left\{\min \left\{\mathbf{N}\left(n_{1}\right), \mu(c)\right\}\right\}\right\} \\
x=a b & \left\{\sup _{x=a b}\{\mu(b)\}, \sup _{x=n_{1}\left(n_{2}+c\right)-n_{1} n_{2}}\{\mu(c)\}\right\} .
\end{aligned}
$$

Let $\sup _{x=a b}\{\mu(a)\}=t_{1}, \sup _{x=a b}\{\mu(b)\}=t_{2}$, and $\sup _{x=n_{1}\left(n_{2}+c\right)-n_{1} n_{2}}\{\mu(c)\}=t_{3}$ for any $a, b$, $n_{1}, n_{2}$, and $c$ in $N$. Assume that $\min \left\{t_{1}, t_{2}, t_{3}\right\}=t_{1}$. Then $a, b, c \in \mu_{t_{1}}$. Since $\mu_{t_{1}}$ is a quasiideal of $N$, then $x=a b \in N \mu_{t_{1}}, x=a b \in \mu_{t_{1}} N$, and $x=n_{1}\left(n_{2}+c\right)-n_{1} n_{2} \in N * \mu_{t_{1}}$. This implies $x \in \mu_{t_{1}} N \cap N \mu_{t_{1}} \cap N * \mu_{t_{1}} \subseteq \mu_{t_{1}}$. Thus, $\mu(x) \geq t_{1}=\min \left\{t_{1}, t_{2}, t_{3}\right\}$. Hence, $(\mu \mathrm{N} \cap$ $\mathbf{N} \mu \cap \mathbf{N} * \mu)(x) \leq t_{1} \leq \mu(x)$. Similarly, if we take $\min \left\{t_{1}, t_{2}, t_{3}\right\}=t_{2}$ or $t_{3}$, we can prove that $(\mu \mathbf{N} \cap \mathbf{N} \mu \cap \mathbf{N} * \mu)(x) \leq t_{2}$ or $t_{3} \leq \mu(x)$. Thus $(\mu \mathbf{N} \cap \mathbf{N} \mu \cap \mathbf{N} * \mu)(x) \leq \mu(x)$, for all $x \in N$. This shows that $\mu$ is a fuzzy quasi-ideal of $N$.

Theorem 2.27. Let $\mu$ be a fuzzy subset of $N$. If $\mu$ is a fuzzy bi-ideal of $N$, if and only if $\mu_{t}$ is a bi-ideal of $N$, for all $t \in \operatorname{Im}(\mu)$.

Proof. Let $\mu$ be a fuzzy bi-ideal of $N$. Let $t \in \operatorname{Im}(\mu)$. Suppose $x, y \in N$ such that $x, y \in$ $\mu_{t}$. Then, $\mu(x) \geq t, \mu(y) \geq t$, and $\min \{\mu(x), \mu(y)\} \geq t$. As $\mu$ is a fuzzy bi-ideal, $\mu(x-$ $y) \geq t$ and thus $x-y \in \mu_{t}$. Let $z \in N$. Suppose $z \in \mu_{t} N \mu_{t} \cap \mu_{t} N * \mu_{t}$. Then there exist $x, y, a_{1}, a_{2}, b \in \mu_{t}$ and $n_{1}, n_{2}, n_{3} \in N$ such that $z=x n_{1} y=a_{1} n_{2}\left(a_{2} n_{3}+b\right)-a_{1} n_{2} a_{2} n_{3}$. Then, $(\mu \mathbf{N} \mu \cap \mu \mathbf{N} * \mu)(z)=\min \{(\mu \mathbf{N} \mu)(z),(\mu \mathbf{N} * \mu)(z)\}$.

Now,

$$
\begin{gathered}
(\mu \mathbf{N} \mu)(z)=\sup _{z=x n_{1} y}\{\min \{\mu(x), \mu(y)\}\} \geq t, \\
(\mu \mathbf{N} * \mu)(z)=\sup _{z=a_{1} n_{2}\left(a_{2} n_{3}+b\right)-a_{1} n_{2} a_{2} n_{3}}\left\{\min \left\{\mu\left(a_{1}\right), \mu(b)\right\}\right\} \geq t .
\end{gathered}
$$

Therefore, $\min \{(\mu \mathbf{N} \mu)(z),(\mu \mathbf{N} * \mu)(z)\} \geq t$ and thus $(\mu \mathbf{N} \mu \cap \mu \mathbf{N} * \mu)(z) \geq t$. As $\mu$ is a biideal of $N, \mu(z) \geq t$ implies $z \in \mu_{t}$. Hence $\mu_{t}$ is a bi-ideal in $N$.

Conversely, let us assume that $\mu_{t}$ is a bi-ideal of $N, t \in \operatorname{Im}(\mu)$. Let $p \in N$. Consider

$$
\begin{aligned}
(\mu \mathbf{N} \mu \cap \mu \mathbf{N} * \mu)(p) & =\min \{(\mu \mathbf{N} \mu)(p),(\mu \mathbf{N} * \mu)(p)\} \\
& =\min \left\{\sup _{p=x n y}\{\min \{\mu(x), \mu(y)\}\}, \sup _{p=a_{1} n_{1}(b+c)-a_{1} n_{1} b}\left\{\min \left\{\mu\left(a_{1}\right), \mu(c)\right\}\right\}\right\} \\
& =\sup _{p=x n y=a_{1} n_{1}(b+c)-a_{1} n_{1} b}\left\{\min \left\{\mu(x), \mu(y), \mu\left(a_{1}\right), \mu(c)\right\}\right\} .
\end{aligned}
$$


Let $\mu(x)=t_{1}<\mu(y)=t_{2}<\mu\left(a_{1}\right)=t_{3}<\mu(c)=t_{4}$. Then, $\mu_{t_{1}} \supseteq \mu_{t_{2}} \supseteq \mu_{t_{3}} \supseteq \mu_{t_{4}}$. Thus, $x, y, a_{1}$, $c \in \mu_{t_{1}}, p=x n y \in \mu_{t_{1}} \mathbf{N} \mu_{t_{1}}$, and $p=a_{1} n_{1}(b+c)-a_{1} n_{1} b \in \mu_{t_{1}} \mathbf{N} * \mu_{t_{1}}$. Thus, $p \in \mu_{t_{1}}$ $\mathbf{N} \mu_{t_{1}} \cap \mu_{t_{1}} \mathbf{N} * \mu_{t_{1}} \subseteq \mu_{t_{1}}$. This implies $\mu(p) \geq t_{1}$ and hence $\mu \mathbf{N} \mu \cap \mu \mathbf{N} * \mu \leq \mu$. Therefore, $\mu$ is a fuzzy bi-ideal of $N$.

\section{Residual quotient fuzzy subset in $N$}

Lemma 3.1. If $\mu$ is a fuzzy left ideal of $N$, then $\mu\left(n_{0} x\right) \geq \mu(x)$ for all $x \in N$ and $n_{0} \in N_{0}$.

Proof. As $\mu$ is a fuzzy left ideal of $N, \mu\left(n_{1}\left(n_{2}+c\right)-n_{1} n_{2}\right) \geq \mu(c)$ for all $n_{1}, n_{2}, c \in N$. Taking $n_{1}=n_{0} \in N_{0}$ and $n_{2}=0$, we have $\mu\left(n_{0} c\right) \geq \mu(c)$.

Lemma 3.2. $N$ is zero-symmetric near-ring if and only if each fuzzy left ideal of $N$ is a fuzzy $N$-subgroup of $N$.

Proof. Assume that $N=N_{0}$. Let $\mu$ be a fuzzy left ideal of $N$. As $\mu$ is a fuzzy left ideal of $N$, by Lemma 3.1, $\mu\left(n_{0} x\right) \geq \mu(x)$ for all $x \in N$ and $n_{0} \in N_{0}=N$. Thus, $\mu$ is a fuzzy $N$ subgroup of $N$.

Conversely, let us assume that each fuzzy left ideal of $N$ is a fuzzy $N$-subgroup of $N$. Let $L$ be a left ideal of $N$. Then, $f_{L}$ is a fuzzy $N$-subgroup of $N$. This implies $f_{L}(n x) \geq f_{L}(x)$ for all $n, x \in N$. In particular, $x \in L$ and $n \in N$, then $N L \subseteq L$. Taking $L$ as $\{0\}$, we have $N\{0\} \subseteq\{0\}$. This implies $N \cdot 0=\{0\}$ and hence $N=N_{0}$.

Now we introduce the notion of residual quotient fuzzy subset $(\lambda: \mu)$ for any two fuzzy subsets $\lambda$ and $\mu$ and annihilator, $\operatorname{ann}(\mu)$, of fuzzy subset $\mu$ of $N$.

Definition 3.3. Let $\lambda$ and $\mu$ be any two fuzzy subsets of $N$. The residual quotient fuzzy $\operatorname{subset}(\lambda: \mu)$ of $N$ is defined as

$$
\begin{aligned}
(\lambda: \mu)(x) & =\vee_{t \in \operatorname{Im}(\lambda)}\left\{t: x \in\left(\lambda_{t}: \mu_{\alpha}\right), \text { where } \alpha=\sup \{\operatorname{Im}(\mu)\}\right\} \\
& =0, \quad \text { otherwise, }
\end{aligned}
$$

where $\left(\lambda_{t}: \mu_{\alpha}\right)=\left\{x \in N \mid x \mu_{\alpha} \subseteq \lambda_{t}\right\}$.

Definition 3.4. Let $O$ be a fuzzy subset defined as $O(0)=1$ and $O(x)=0$ for all $x \neq 0 \in N$. Then $(O: \mu)$ is the annihilator of $\mu$ and it is denoted by $\operatorname{ann}(\mu)$.

Definition 3.4 is different from the definition for $\operatorname{ann}(\mu)$ given in [12]. It is clear that, for any $t_{1}, t_{2} \in \operatorname{Im}(\lambda)$ and $\alpha=\sup \{\operatorname{Im}(\mu)\}$ with $t_{1}<t_{2}$, we have $\left(\lambda_{t_{2}}: \mu_{\alpha}\right) \subseteq\left(\lambda_{t_{1}}: \mu_{\alpha}\right)$. For, let $x \in\left(\lambda_{t_{2}}: \mu_{\alpha}\right)$. Then, $x \mu_{\alpha} \subseteq \lambda_{t_{2}} \subseteq \lambda_{t_{1}}$. Thus, $x \in\left(\lambda_{t_{1}}: \mu_{\alpha}\right)$ and therefore $\left(\lambda_{t_{2}}: \mu_{\alpha}\right) \subseteq\left(\lambda_{t_{1}}\right.$ : $\left.\mu_{\alpha}\right)$.

Theorem 3.5. Let $\lambda$ and $\mu$ be any two fuzzy subsets of $N$. If $\lambda$ is a fuzzy left ideal of $N$, then $(\lambda: \mu)$ is a fuzzy left ideal of $N$.

Proof. Let $x, y \in N$ and $\alpha=\sup \{\operatorname{Im}(\mu)\}$. Suppose $(\lambda: \mu)(x)=t_{1}$ and $(\lambda: \mu)(y)=t_{2}$, where $t_{1}, t_{2} \neq 0 \in \operatorname{Im}(\lambda)$. Assume that $t_{1}<t_{2}$. Then, $\left(\lambda_{t_{2}}: \mu_{\alpha}\right) \subseteq\left(\lambda_{t_{1}}: \mu_{\alpha}\right)$. Thus, $x, y \in\left(\lambda_{t_{1}}: \mu_{\alpha}\right)$ implies for any $b \in \mu_{\alpha}$; we have $(x-y) b=x b-y b \in \lambda_{t_{1}}$. Then, $(x-y) \mu_{\alpha} \subseteq \lambda_{t_{1}}$. Hence 
$(x-y) \in\left(\lambda_{t_{1}}: \mu_{\alpha}\right)$ which implies that $(\lambda: \mu)(x-y) \geq t_{1}$ and $(\lambda: \mu)(x-y) \geq t_{1}=\min \left\{t_{1}\right.$, $\left.t_{2}\right\}$. Similarly, if $t_{1}>t_{2}$, then $(\lambda: \mu)(x-y) \geq t_{2}=\min \left\{t_{1}, t_{2}\right\}$. Thus, $(\lambda: \mu)(x-y) \geq$ $\min \{(\lambda: \mu)(x),(\lambda: \mu)(y)\}$. For other choices of $t_{1}$ and $t_{2}$, it can be verified that $(\lambda: \mu)(x-$ $y) \geq \min \{(\lambda: \mu)(x),(\lambda: \mu)(y)\}$. Suppose $(\lambda: \mu)(x+y)=t$. Then, $x+y \in\left(\lambda_{t}: \mu_{\alpha}\right)$. As $\lambda_{t}$ is normal subgroup of $N,\left(\lambda_{t}: \mu_{\alpha}\right)$ is also normal subgroup of $N$. Then, $y+x \in\left(\lambda_{t}: \mu_{\alpha}\right)$. Thus, $(\lambda: \mu)(y+x) \geq t=(\lambda: \mu)(x+y)$. Similarly, $(\lambda: \mu)(x+y) \geq(\lambda: \mu)(y+x)$. Hence, $(\lambda: \mu)(x+y)=(\lambda: \mu)(y+x)$. Let $(\lambda: \mu)(x)=t$. Now, $x \in\left(\lambda_{t}: \mu_{\alpha}\right)$. Thus for any $a, b \in N$, $a(b+x)-a b \in\left(\lambda_{t}: \mu_{\alpha}\right)$. This implies $(\lambda: \mu)(a(b+x)-a b) \geq t=(\lambda: \mu)(x)$. Thus $(\lambda: \mu)$ is a fuzzy left ideal of $N$.

Corollary 3.6. Let $\mu$ be any fuzzy subset of $N$, then $\operatorname{ann}(\mu)$ is a fuzzy left ideal of $N$.

Remark 3.7. Let $\lambda$ and $\mu$ be any two fuzzy subsets of $N$. If $\lambda$ is a fuzzy left ideal of $N$, then $(\lambda: \mu)$ is not necessarily fuzzy ideal of $N$ as the following example shows.

Example 3.8. Let $N=\{0, a, b, c\}$ be Klein's four group. Define multiplication in $N$ as follows:

\begin{tabular}{c|cccc}
\hline+ & 0 & $\mathrm{a}$ & $\mathrm{b}$ & $\mathrm{c}$ \\
\hline 0 & 0 & $\mathrm{a}$ & $\mathrm{b}$ & $\mathrm{c}$ \\
$\mathrm{a}$ & $\mathrm{a}$ & 0 & $\mathrm{c}$ & $\mathrm{b}$ \\
$\mathrm{b}$ & $\mathrm{b}$ & $\mathrm{c}$ & 0 & $\mathrm{a}$ \\
$\mathrm{c}$ & $\mathrm{c}$ & $\mathrm{b}$ & $\mathrm{a}$ & 0 \\
\hline
\end{tabular}

\begin{tabular}{l|llll}
\hline$\bullet$ & 0 & $\mathrm{a}$ & $\mathrm{b}$ & $\mathrm{c}$ \\
\hline 0 & 0 & 0 & 0 & 0 \\
$\mathrm{a}$ & $\mathrm{a}$ & $\mathrm{a}$ & $\mathrm{a}$ & $\mathrm{a}$ \\
$\mathrm{b}$ & 0 & $\mathrm{a}$ & $\mathrm{b}$ & $\mathrm{c}$ \\
$\mathrm{c}$ & $\mathrm{a}$ & 0 & $\mathrm{c}$ & $\mathrm{b}$ \\
\hline
\end{tabular}

Then $(N,+, \bullet)$ is a near-ring (see $[9$, Page 408 , Scheme 20]). Let $\lambda$ be a fuzzy subset of $N$. Define $\lambda: N \rightarrow[0,1]$ by $\lambda(0)=\lambda(b)=1$ and $\lambda(a)=\lambda(c)=0$. For any $t \in[0,1]$, we have only two level sets, viz, $\{0, b\}$ and $\{0, a, b, c\}$. Since $\{0, b\}$ is the zero-symmetric part of $N$, by [9, Proposition 1.32(a)], $\{0, b\}$ is a left ideal in $N$. By Lemma 2.16, $\lambda$ is a fuzzy left ideal of $N$. Let $\mu$ be any fuzzy subset of $N$. Define $\mu: N \rightarrow[0,1]$ by $\mu(c)=1$ and $\mu(0)=\mu(a)=\mu(b)=0$. Now we have $(\lambda: \mu)(0)=1,(\lambda: \mu)(a)=0,(\lambda: \mu)(b)=0$, and $(\lambda: \mu)(c)=1$, as $(\lambda: \mu)(c . c)=(\lambda: \mu)(b)=0 \nsupseteq(\lambda: \mu)(c)$. Hence, $(\lambda: \mu)$ is not a fuzzy ideal of $N$.

Now we find the conditions under which $(\lambda: \mu)$ is a fuzzy ideal of $N$.

Theorem 3.9. Let $\lambda$ and $\mu$ be any two fuzzy subsets of $N$. If $\lambda$ is a fuzzy left ideal and $\mu$ is a fuzzy $N$-subgroup of $N$, then $(\lambda: \mu)$ is a fuzzy ideal of $N$.

Proof. By Theorem 3.5, $(\lambda: \mu)$ is a fuzzy left ideal of $N$. Let $(\lambda: \mu)(x)=t$. Then, $x \in\left(\lambda_{t}\right.$ : $\left.\mu_{\alpha}\right)$, where $\alpha=\sup \{\operatorname{Im}(\mu)\}$. Now, $x \mu_{\alpha} \subseteq \lambda_{t}$. Let $n \in N$. Consider $x n b=x(n b) \in x \mu_{\alpha} \subseteq \lambda_{t}$. Since $\mu_{\alpha}$ is $N$-subgroup of $N, n b \in \mu_{\alpha}$ for all $n \in N$ and $b \in \mu_{\alpha}, x n \mu_{\alpha} \subseteq \lambda_{t}$. This implies $x n \in\left(\lambda_{t}: \mu_{\alpha}\right)$. Thus $(\lambda: \mu)(x n) \geq t=(\lambda: \mu)(x)$. Hence, $(\lambda: \mu)$ is a fuzzy right ideal of $N$. Therefore, $(\lambda: \mu)$ is a fuzzy ideal of $N$. 
Example 3.10. Let $N=\{0, a, b, c\}$ be Klein's four group. Define multiplication in $N$ as follows:

\begin{tabular}{c|cccc}
\hline+ & 0 & $\mathrm{a}$ & $\mathrm{b}$ & $\mathrm{c}$ \\
\hline 0 & 0 & $\mathrm{a}$ & $\mathrm{b}$ & $\mathrm{c}$ \\
$\mathrm{a}$ & $\mathrm{a}$ & 0 & $\mathrm{c}$ & $\mathrm{b}$ \\
$\mathrm{b}$ & $\mathrm{b}$ & $\mathrm{c}$ & 0 & $\mathrm{a}$ \\
$\mathrm{c}$ & $\mathrm{c}$ & $\mathrm{b}$ & $\mathrm{a}$ & 0 \\
\hline
\end{tabular}

\begin{tabular}{c|cccc}
\hline$\bullet$ & 0 & $\mathrm{a}$ & $\mathrm{b}$ & $\mathrm{c}$ \\
\hline 0 & 0 & 0 & 0 & 0 \\
$\mathrm{a}$ & 0 & $\mathrm{a}$ & $\mathrm{b}$ & $\mathrm{c}$ \\
$\mathrm{b}$ & 0 & 0 & 0 & 0 \\
$\mathrm{c}$ & 0 & $\mathrm{a}$ & $\mathrm{b}$ & $\mathrm{c}$ \\
\hline
\end{tabular}

Then $(N,+, \bullet)$ is a near-ring (see [9, Page 408, Scheme 13]).

Define $\lambda: N \rightarrow[0,1]$ by

$$
\lambda(x)= \begin{cases}1, & \text { if } x=0, a \\ 0, & \text { otherwise }\end{cases}
$$

Clearly $\lambda$ is a fuzzy left ideal of $N$. But $\lambda$ is not a fuzzy right ideal of $N$, since $0=\lambda(a c) \nsupseteq$ $\lambda(a)=1$. Define $\mu: N \rightarrow[0,1]$ by

$$
\mu(x)= \begin{cases}1, & x=0, c \\ 0, & \text { otherwise }\end{cases}
$$

Clearly $\mu$ is a fuzzy $N$-subgroup of $N$. Now for any $x \in N$,

$$
\begin{aligned}
(\lambda: \mu)(x)=1 & \Longleftrightarrow x \in\left(\lambda_{1}: \mu_{1}\right) \\
& \Longleftrightarrow x \in(\{0, a\}:\{0, c\}) \\
& \Longleftrightarrow x \in\{0, b\} .
\end{aligned}
$$

Thus, $(\lambda: \mu)(0)=(\lambda: \mu)(b)=1$ and $(\lambda: \mu)(a)=(\lambda: \mu)(c)=0$. Hence, $(\lambda: \mu)$ is a fuzzy ideal of $N$. Note that $\lambda$ is only a fuzzy left ideal but not a fuzzy ideal of $N$. Now, $\operatorname{ann}(\mu)(0)=\operatorname{ann}(\mu)(b)=1$ and $\operatorname{ann}(\mu)(a)=\operatorname{ann}(\mu)(c)=0$. Clearly $\operatorname{ann}(\mu)$ is a fuzzy ideal of $N$.

Corollary 3.11. If $\mu$ is a fuzzy $N$-subgroup of $N$, then $\operatorname{ann}(\mu)$ is a fuzzy ideal of $N$.

Theorem 3.12. Let $\lambda$ and $\mu$ be any two fuzzy subset of $N$. If $\lambda$ and $\mu$ are fuzzy ideals of zero-symmetric near-ring $N$, then $(\lambda: \mu)$ is a fuzzy ideal of $N$.

Proof. $(\lambda: \mu)$ is a fuzzy left ideal of $N$, by Theorem 3.5. Now $\mu$ is a fuzzy ideal of $N$, then by Lemma 3.2, $\mu$ is a fuzzy $N$-subgroup of $N$. Thus by the Theorem 3.9, $(\lambda: \mu)$ is a fuzzy ideal of $N$.

Lemma 3.13. Let $\lambda$ be a fuzzy ideal of $N$. $\lambda$ is completely fuzzy semiprime ideal of $N$ if and only if $\lambda_{t}, t \in \operatorname{Im}(\lambda)$ is a completely semiprime ideal of $N$. 
Proof. Let $\lambda$ be a fuzzy completely semiprime ideal of $N$. Let $t \neq 0 \in \operatorname{Im}(\lambda)$. Let $x \in N$ such that $x^{2} \in \lambda_{t}$. This implies $\left(x_{t}\right)^{2} \in \lambda$. As $\lambda$ is a fuzzy completely semiprime ideal of $N$, $x_{t} \in \lambda$. This implies $x \in \lambda_{t}$.

Conversely, let us assume that $\lambda_{t}$ is a completely semiprime ideal of $N, t \in \operatorname{Im}(\lambda)$. Suppose $y_{s}^{2} \in \lambda$. Then, $y^{2} \in \lambda_{s}$ and $y_{s} \in \lambda$. Thus, $\lambda$ is a fuzzy completely semiprime ideal of $N$.

Theorem 3.14. Let $\lambda$ be a fuzzy ideal of $N$. If $a_{r} b_{s} \in \lambda$ implies $b_{s} a_{r} \in \lambda$ for any fuzzy points $a_{r}, b_{s}$ of $N$, then for any fuzzy subset $\mu$ of $N,(\lambda: \mu)$ is a fuzzy ideal of $N$.

Proof. By Theorem 3.5, $(\lambda: \mu)$ is a fuzzy left ideal. Let $(\lambda: \mu)(x)=t$ and $\alpha=\sup \{\operatorname{Im}(\mu)\}$. This implies that $x \in\left(\lambda_{t}: \mu_{\alpha}\right)$ and $x b \in \lambda_{t}$, for all $b \in \mu_{\alpha}$. Then, $(x b)_{t}=x_{t} b_{t}=b_{t} x_{t} \in \lambda$. Hence, $b x \in \lambda_{t}$. As $\lambda_{t}$ is an ideal of $N, n b x \in \lambda_{t}$ and hence $(n b x)_{t} \in \lambda$, for all $n \in N$. Now, $(x n b)_{t}=x_{t}\left(n_{t} b_{t}\right)=\left(n_{t} b_{t}\right) x_{t}=(n b x)_{t} \in \lambda$ and hence $x n b \in \lambda_{t}$. This implies that $x n \in\left(\mu_{t}\right.$ : $\left.\mu_{\alpha}\right)$. Thus, $(\lambda: \mu)(x n) \geq t=(\lambda: \mu)(x)$. Hence, $(\lambda: \mu)$ is a fuzzy right ideal of $N$. Therefore, $(\lambda: \mu)$ is a fuzzy ideal of $N$.

THeOREM 3.15. Let $\lambda$ and $\mu$ be any fuzzy subsets of $N$. If $\lambda$ is a fuzzy completely semiprime ideal of $N$, then $(\lambda: \mu)$ is a fuzzy ideal of $N$.

Proof. By Theorem 3.5, $(\lambda: \mu)$ is a fuzzy left ideal of $N$. Let $(\lambda: \mu)(x)=t$ and $\alpha=\sup \{\operatorname{Im}$ $(\mu)\}$. Then, $x \in\left(\lambda_{t}: \mu_{\alpha}\right)$. This implies $x \mu_{\alpha} \subseteq \lambda_{t}$. For any $n \in N$, let us show that $x n \in$ $\left(\lambda_{t}: \mu_{\alpha}\right)$. As $x \mu_{\alpha} \subseteq \lambda_{t}, x b \in \lambda_{t}$ for all $b \in \mu_{\alpha}$. Now, $(b x)^{2}=b x b x=b(x b) x \in \lambda_{t}$. As $\lambda_{t}$ is a completely semiprime ideal in $N, b x \in \lambda_{t}$. Consider $(x n b)^{2}=x n b x n b=x n(b x) n b \in \lambda_{t}$. This implies $x n b \in \lambda_{t}$ for every $b \in \mu_{t}$. Thus, $x n \in\left(\lambda_{t}: \mu_{\alpha}\right)$. Hence, $(\lambda: \mu)(x n)>t=(\lambda$ : $\mu)(x)$. Therefore, $(\lambda: \mu)$ is a fuzzy ideal of $N$.

Theorem 3.16. If $\lambda$ is a fuzzy bi-ideal and let $\mu$ is a fuzzy $N$-subgroup of zero-symmetric near-ring $N$, then $(\lambda: \mu)$ is a fuzzy bi-ideal of $N$.

Proof. Let $\lambda$ be a fuzzy bi-ideal and $\mu$ be a fuzzy $N$-subgroup of zero-symmetric near-ring $N$. Clearly $(\lambda: \mu)$ is a fuzzy subgroup of $N$. Next we prove that $(\lambda: \mu)$ is a fuzzy bi-ideal of $N$. Let $t \in N$ and $a, n, b \in N$ such that $t=$ anb. Consider

$$
\begin{aligned}
((\lambda: \mu) \mathbf{N}(\lambda: \mu))(t) & =\sup _{t=\text { anb }}\{\min \{(\lambda: \mu)(a), \mathbf{N}(n),(\lambda: \mu)(b)\}\} \\
& =\sup _{t=\text { anb }}\{\min \{(\lambda: \mu)(a),(\lambda: \mu)(b)\}\} .
\end{aligned}
$$

Let $\min \{(\lambda: \mu)(a),(\lambda: \mu)(b)\}=t$. This implies that $(\lambda: \mu)(a) \geq t$ and $(\lambda: \mu)(b) \geq t$. Then, $a, b \in\left(\lambda_{t}: \mu_{\alpha}\right)$. As $\lambda$ is the fuzzy bi-ideal and $\mu$ is the fuzzy $N$-subgroup, $\left(\lambda_{t}: \mu_{\alpha}\right)$ is a bi-ideal of $N$. Hence, anb $\in\left(\lambda_{t}: \mu_{\alpha}\right)$. This implies $(\lambda: \mu)(\mathrm{anb}) \geq t=\min \{(\lambda: \mu)(a),(\lambda$ : $\mu)(b)\}$. Thus, $\min \{(\lambda: \mu)(a),(\lambda: \mu)(b)\} \leq(\lambda: \mu)(\mathrm{anb})$. This shows that $\sup _{t=\mathrm{anb}} \min \{(\lambda:$ $\mu)(a),(\lambda: \mu)(b)\} \leq(\lambda: \mu)($ anb $)$. Thus, we have $((\lambda: \mu) \mathbf{N}(\lambda: \mu))(t) \leq(\lambda: \mu)(t)$. Hence, $(\lambda$ : $\mu) \mathbf{N}(\lambda: \mu) \leq(\lambda: \mu)$ and $(\lambda: \mu)$ is a fuzzy bi-ideal of $N$. 


\section{Residual quotient fuzzy subset in near-rings}

\section{References}

[1] S. Abou-Zaid, On fuzzy subnear-rings and ideals, Fuzzy Sets and Systems 44 (1991), no. 1, 139146.

[2] P. S. Das, Fuzzy groups and level subgroups, Journal of Mathematical Analysis and Applications 84 (1981), no. 1, 264-269.

[3] V. N. Dixit, R. Kumar, and N. Ajmal, Fuzzy ideals and fuzzy prime ideals of a ring, Fuzzy Sets and Systems 44 (1991), no. 1, 127-138.

[4] R. Kumar, Fuzzy Algebra Volume I, University of Delhi Publication Division, Delhi, 1993.

[5] W. J. Liu, Fuzzy invariant subgroups and fuzzy ideals, Fuzzy Sets and Systems 8 (1982), no. 2, $133-139$.

[6] __ Operations on fuzzy ideals, Fuzzy Sets and Systems 11 (1983), no. 1, 31-41.

[7] T. K. Mukherjee and M. K. Sen, Prime fuzzy ideals in rings, Fuzzy Sets and Systems 32 (1989), no. 3, 337-341.

[8] AL. Narayanan, Contributions to the algebraic structures in fuzzy theory, Ph.D. thesis, Annamalai University, Tamil Nadu, 2001.

[9] G. Pilz, Near-Rings, 2nd ed., North-Holland Mathematics Studies, vol. 23, North-Holland, Amsterdam, 1983.

[10] A. Rosenfeld, Fuzzy groups, Journal of Mathematical Analysis and Applications 35 (1971), no. 3, 512-517.

[11] H. K. Saikia and L. K. Barthakur, On fuzzy N-subgroups and fuzzy ideals of near-rings and nearring groups, The Journal of Fuzzy Mathematics 11 (2003), no. 3, 567-580.

[12] _ Characterization of fuzzy substructures of a near-ring and a near-ring group, The Journal of Fuzzy Mathematics 13 (2005), no. 1, 159-167.

[13] U. M. Swamy and K. L. N. Swamy, Fuzzy prime ideals of rings, Journal of Mathematical Analysis and Applications 134 (1988), no. 1, 94-103.

[14] L. A. Zadeh, Fuzzy sets, Information and Control 8 (1965), 338-353.

P. Dheena: Department of Mathematics, Annamalai University, Annamalai Nagar 608002, India E-mail address: dheenap@yahoo.com

S. Coumaressane: Department of Mathematics, Annamalai University, Annamalai Nagar 608002, India

E-mail address: coumaressane_s@yahoo.com 


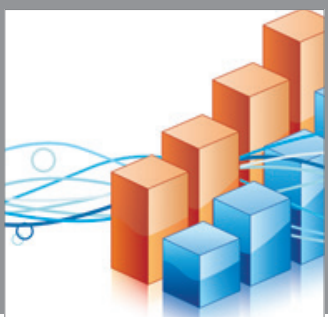

Advances in

Operations Research

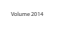

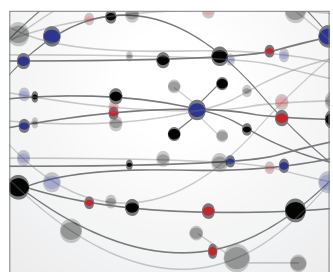

\section{The Scientific} World Journal
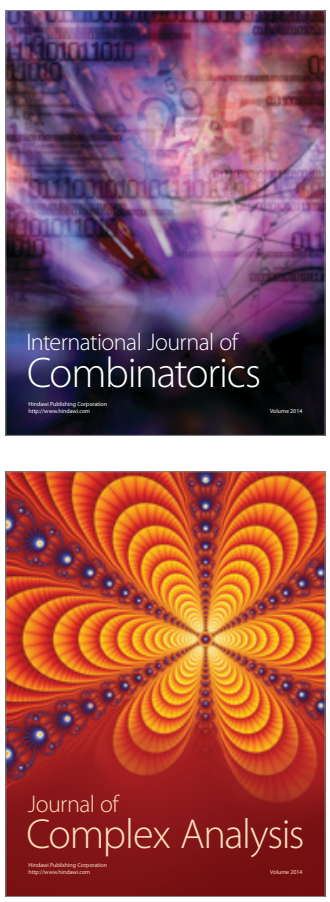

International Journal of

Mathematics and

Mathematical

Sciences
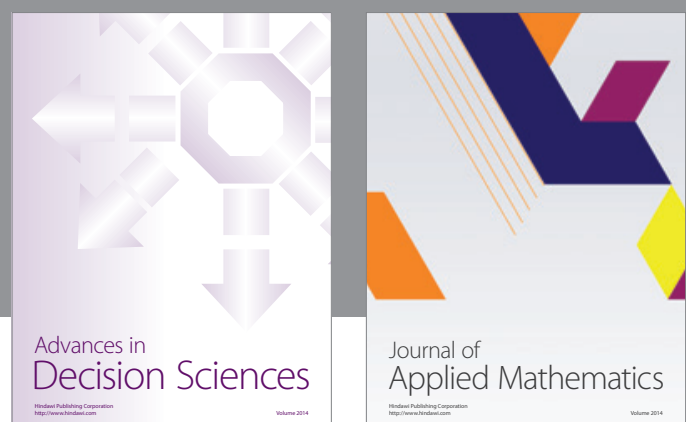

Journal of

Applied Mathematics
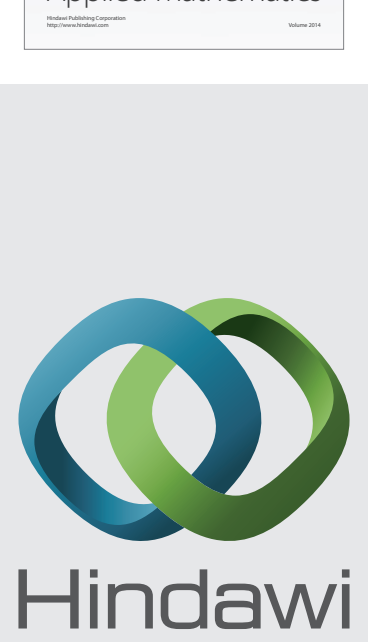

Submit your manuscripts at http://www.hindawi.com
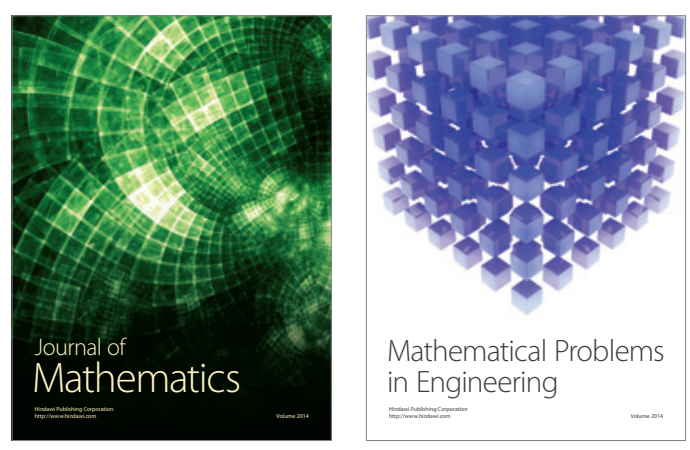

Mathematical Problems in Engineering
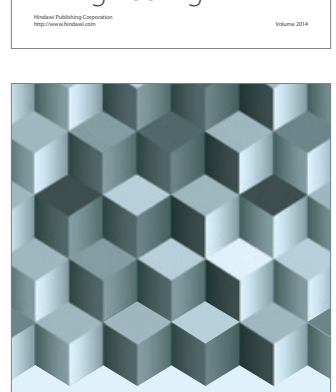

Journal of

Function Spaces
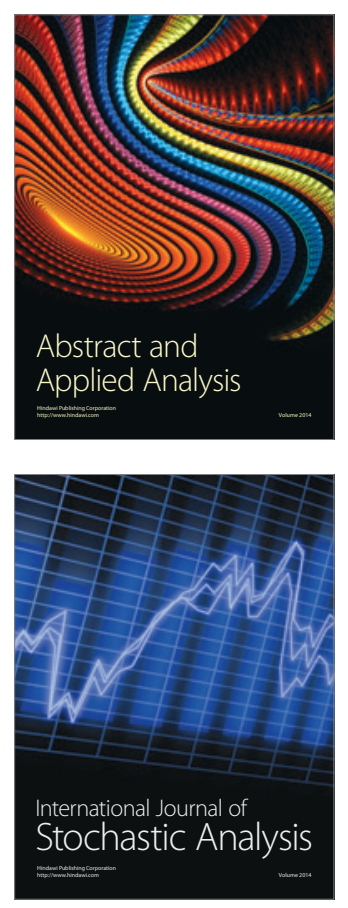

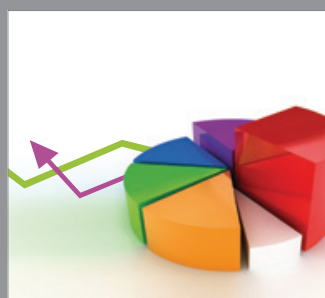

ournal of

Probability and Statistics

Promensencen
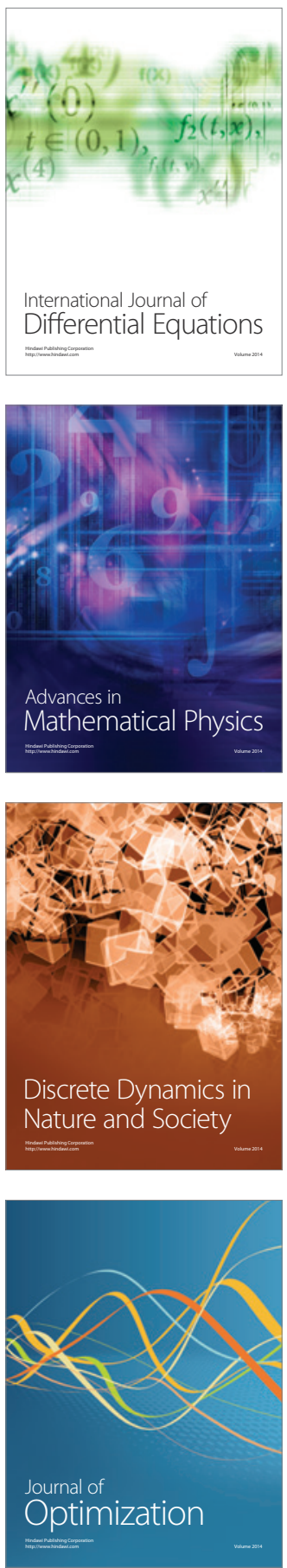